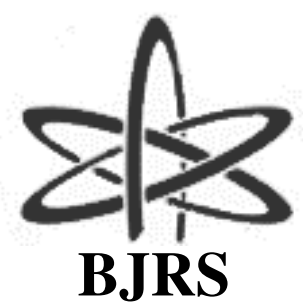

BRAZILIAN JOURNAL

$\mathrm{OF}$

RADIATION SCIENCES

03-1A (2015) 01-08

\title{
Sistema de instrumentação de multimedição de parâmetros de equipamentos de raios $x$ diagnósticos
}

\author{
Luiz A. P. Santos ${ }^{1}$, David S. Monte ${ }^{1}$, José A. P. Santos ${ }^{1}$, Francisco A. Cavalcanti ${ }^{2}$, Fábio \\ R. Barros ${ }^{2}$ e Marcus A. P. Santos ${ }^{2}$ \\ ${ }^{1}$ SCIENTS \\ Av. Professor Luiz Freire, $n^{\circ} 700-C D U$ \\ 50740-540 Recife, PE \\ lasantos@scients.com.br \\ ${ }^{2}$ Centro Regional de Ciências Nucleares do Nordeste (CRCN-NE) \\ Comissão Nacional de Energia Nuclear (CNEN) \\ Av. Professor Luiz Freire, $n^{o} 1$ - CDU \\ 50740-540 Recife, PE \\ frbarros@cnen.gov.br
}

\begin{abstract}
RESUMO
As diretrizes básicas de proteção radiológica em diagnóstico médico e odontológico do Ministério da Saúde publicada em 1998 seguiu as recomendações da Comissão Nacional de Energia Nuclear (CNEN) que tem como base as recomendações da Comissão Internacional de Proteção Radiológica (ICRP), da Organização Mundial de Saúde (WHO) e também da Agência Internacional de Energia Atômica (IAEA). Essas diretrizes consistem em um regulamento técnico que estabelecem requisitos básicos de proteção radiológica em radiodiagnóstico e disciplina a prática com os raios-X para fins diagnósticos e intervencionistas, visando a defesa da saúde dos pacientes, dos profissionais envolvidos e do público em geral. Parte desta norma determina que todo equipamento de raios-X diagnóstico deve ser mantido em condições adequadas de funcionamento e submetido regularmente a verificações de desempenho, ou seja, deve-se verificar a conformidade dos principais parâmetros envolvidos em testes periódicos para se garantir um controle de qualidade dos serviços prestados. A exatidão da tensão de tubo ( $\mathrm{kVp}$ ) e do tempo de exposição, o valor da Camada Semi-Redutora (CSR), e a reprodutibilidade da taxa de kerma são alguns parâmetros ou grandezas que devem ser mensurados em equipamentos convencionais de raios X diagnósticos, enquanto que o tamanho de campo de radiação e o número de pulsos são outros parâmetros associados aos equipamentos de raios $\mathrm{X}$ odontológicos. Na realidade, nem sempre é possível realizar um procedimento de medição para várias grandezas simultaneamente e isso fez com que nos últimos cinco anos diversas instituições internacionais desenvolvessem instrumentação para atender esse tipo de necessidade. Nesta comunicação, pretendese apresentar metodologias inovadoras de um sistema de instrumentação de multi-medição dos seguintes parâmetros: CSR, kVp, kerma no ar, taxa de kerma, tempo de exposição, tamanho de campo e o número de pulsos de raios X. Assim, o instrumento se aplica tanto para mensurar alguns parâmetros de equipamentos de raios $\mathrm{X}$ convencionais como de equipamentos odontológicos. As metodologias utilizadas são baseadas em modernas técnicas eletrônicas de medição com sensores de raios $\mathrm{X}$ não convencionais, como o transistor bipolar de junção (TBJ) polarizado com determinados sinais elétricos. De fato, a polarização adequada permite mensurar cada parâmetro ou grandeza a partir da interferência dos fótons no dispositivo convertendo o sinal de saída do TBJ em um sinal modulado pela interação da radiação. O sinal de saída é então tratado por um microprocessador para ser
\end{abstract}


convertido na grandeza ou parâmetro de avaliação do equipamento de raios-X. Os resultados mostram que o sistema multi-medição proposto apresenta uma incerteza relativa $1 \%$ e 5\% para os parâmetros $\mathrm{kVp}$ e CSR, respectivamente, para os equipamentos de radiografia convencionais, e de menos que $1 \%$ para o tempo de exposição em equipamentos de raios $\mathrm{X}$ odontológicos. $\mathrm{O}$ sistema proposto traz inovação ao estado da técnica e tem caráter metrológico proporcionando ser aplicado ao controle de qualidade de equipamentos de raios X diagnósticos.

\section{INTRODUÇÃO}

O Ministério da Saúde em conjunto com a Comissão Nacional de Energia Nuclear (CNEN) publicaram as diretrizes básicas de proteção radiológica em diagnóstico médico e odontológico [1] as quais na realidade seguem as recomendações da Comissão Internacional de Proteção Radiológica (ICRP), da Organização Mundial de Saúde (WHO) e também da Agência Internacional de Energia Atômica (IAEA). Essas diretrizes consistem em um regulamento técnico que estabelecem requisitos básicos de proteção radiológica em radiodiagnóstico e disciplina a prática com os raios- $X$ tanto para fins de diagnose como também intervencionista, visando a defesa da saúde dos pacientes, dos profissionais envolvidos e do público em geral. A norma, na prática, determina que todo equipamento de raios-X diagnóstico deve ser mantido em condições de funcionamento padronizadas e têm que ser submetidos regularmente a verificações de desempenho, ou seja, deve-se verificar a conformidade dos principais parâmetros envolvidos com testes periódicos para se garantir um controle de qualidade dos serviços prestados. Os principais parâmetros os quais devem ser verificados são: tensão de pico $(\mathrm{kVp})$, camada semiredutora (CSR), kerma no ar, taxa de kerma, tempo de exposição, tamanho de campo e o número de pulsos de raios $\mathrm{X}$. Os dois últimos parâmetros são relativos aos feixes de raios $\mathrm{X}$ de equipamentos utilizados em odontologia. Na realidade, nem sempre é possível realizar procedimentos de medição para várias grandezas simultaneamente e isso fez com que nos últimos cinco anos diversas instituições internacionais desenvolvessem instrumentação para atender essa necessidade. Nesta comunicação, pretende-se apresentar algumas metodologias inovadoras de um sistema de instrumentação de multi-medição dos parâmetros supra citados. Assim, o instrumento se aplica tanto para mensurar alguns parâmetros de equipamentos convencionais de raios $\mathrm{X}$ como também dos equipamentos odontológicos.

Normalmente se utiliza alguns dispositivos eletrônicos semicondutores para atuarem como detectores de fótons de raios $\mathrm{X}$ como por exemplo o fotodiodo ou fototransistor [2-3], os quais estão presentes dentro dos modernos medidores eletrônicos lançados recentemente ou mesmo dentro dos modernos tomógrafos. Em geral, esses dispositivos fornecem um sinal de corrente elétrica que é proporcional a taxa de kerma no ponto de avaliação, na superfície de entrada de um fantôma ou no seu interior, por exemplo. A corrente elétrica $\boldsymbol{i}(t)$ produzida pelo detector é integrado no tempo para se obter via fator de conversão o valor do kerma no ar. Contudo, mensurar outras grandezas ou parâmetros relacionados ao feixe de radiação não são simples, e um método inovador utilizando o transistor bipolar de junção (TBJ) foi apresentado recentemente [4] com propósito de medir a tensão de pico $(\mathrm{kV})$ de equipamentos de raios $\mathrm{X}$ diagnósticos. Neste artigo é apresentado outros métodos que também utiliza o TBJ para medição de alguns parâmetros acima citados.

\section{MATERIAIS E MÉTODOS}




\subsection{Princípio de funcionamento do TBJ}

O transistor bipolar de junção basicamente consiste de duas junções $P N$ de materiais semicondutores podendo ser, por exemplo, NPN ou PNP. O eletrodo denominado de base (B) é um semicondutor tipo $\mathrm{P}(\mathrm{N})$ sanduichado entre dois semicondutores tipo $\mathrm{N}(\mathrm{P})$ denominado coletor (C) e emissor (E). Na Figura 1 está ilustrado o TBJ do tipo NPN bem como uma das formas de ser polarizado: uma fonte de corrente denominada de $\mathrm{I}_{\mathrm{B}}$ é aplicada na junção baseemissor e outra fonte de tensão denominada de $\mathrm{V}_{\mathrm{CE}}$, conhecida como ponto de operação do TBJ, é aplicada entre o coletor e o emissor [5]. Normalmente, a corrente de base $\mathrm{I}_{\mathrm{B}}$ é o sinal de entrada e a corrente de coletor $I_{C}$ é o sinal de saída. Em geral o TBJ é utilizado em circuitos eletrônicos como amplificador e o fator de amplificação do transistor é a relação $\mathrm{I}_{C} / \mathrm{I}_{\mathrm{B}}$ o qual também é denominado de ganho do transistor, $\beta$. Assim, o sinal de saída é dado por:

$$
\mathrm{I}_{\mathrm{C}}=\beta \cdot \mathrm{I}_{\mathrm{B}}
$$
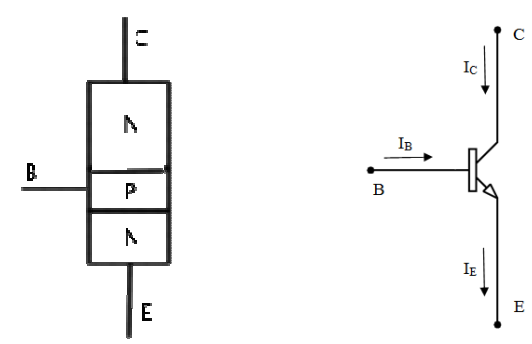

\section{Figura 1. Estrutura de um transistor bipolar de junção NPN e seu símbolo em circuitos eletrônicos.}

\subsection{TBJ funcionando como detector de raios $X$}

O transistor bipolar de junção já foi utilizado como sensor de radiação e de partículas [6], como raios gama, prótons, nêutrons etc., para verificar como é o comportamento da curva característica $\mathrm{I}_{\mathrm{C}} \times \mathrm{V}_{\mathrm{CE}}$ em função da dose de radiação. Recentemente, tem sido usado como um detector de radiação [4] para correlacionar características de feixes de radiação ionizante utilizados em diagnóstico, sendo a perda de sensibilidade não significativa e por isto tal efeito pode então ser negligenciado. Para fazer medições em tempo real com um TBJ, sob um feixe de raios-X, uma baixa intensidade de corrente $\mathrm{I}_{\mathrm{B}}$ (da ordem de dezenas de $\mathrm{pA}$ ) deve ser injetada na base, como sugerido por [4]. Este tipo de polarização proporciona que os fótons de raios $\mathrm{X}$ sensibilizem fortemente o TBJ e por isso a corrente de coletor durante a irradiação, denominada neste artigo de $I_{C X}$, tem um valor significativamente maior que $I_{C}$. Conforme apresentado em [4], caso o sinal $\mathrm{I}_{\mathrm{B}}$ não seja da ordem de picoampere o efeito dos fótons de raios $\mathrm{X}$ diagnósticos praticamente não sensibiliza o TBJ. Assim, é possível avaliar a variação da corrente de coletor $\left(\Delta \mathrm{I}_{\mathrm{C}}=\mathrm{I}_{\mathrm{CX}}-\mathrm{I}_{\mathrm{C}}\right.$ ou $\partial \mathrm{I}_{\mathrm{C}}=\mathrm{I}_{\mathrm{CX}} / \mathrm{I}_{\mathrm{C}}$ ) e correlacionar com um parâmetro a ser mensurado do equipamento de raios $\mathrm{X}$. Na realidade, com $\Delta \mathrm{I}_{\mathrm{CX}}$ ou $\partial \mathrm{I}_{\mathrm{C}}$ é feita uma modulação eletrônica analógica por um sinal digital de 
extrema complexidade cujo detalhe técnico foge ao propósito desta comunicação, além de consistir em sigilo de patente da SCIENTS.

\subsection{Instrumentos eletrônicos e transistores}

A Figura 2 ilustra como deve ser polarizado um TBJ para que ele funcione como detector de fótons de raios $\mathrm{X}$ diagnósticos. Basicamente deve-se utilizar uma fonte de corrente para fornecer $I_{B}$ e outra fonte de tensão para aplicar o ponto de operação $V_{C E}=5 \mathrm{~V}$. A característica da fonte de alimentação $\mathrm{V}_{\mathrm{CE}}$ é do tipo fonte-medidor (source-meter) cuja propriedade é que tal sistema mede simultaneamente a corrente de coletor, $I_{C}$. Tanto $I_{B}$ como $V_{C E}$ (as fontes de polarização) correspondem aos circuitos internos do instrumento RXM1407, marca SCIENTS, cujo detalhe técnico do circuito não pode ser apresentado aqui devido ao sigilo de patente. Para fins de comparação com os resultados do RXM1407 foram utilizados outros dois sistemas de medição: 1) instrumento eletrônico ThinX, marca UNFORS; 2) câmara de ionização 10X5-6, marca RADCAL.

Na prática, há diversos transistores bipolares ( 60 mil) que possibilitam ser utilizados como sensor de fótons de raios $\mathrm{X}$ pois todos têm o comportamento idêntico e basicamente o que muda é o ganho $\beta$ e o encapsulamento. Com relação ao ganho do TBJ, pode-se dizer que quanto maior mais forte será o sinal de saída. Em relação ao encapsulamento não há uma interferência muito significativa [4]. O sistema de multi-medição RXM1407 utiliza cerca de 7 dispositivos internamente, entre eles o BC547, o 2N3904, e o ZTX603.
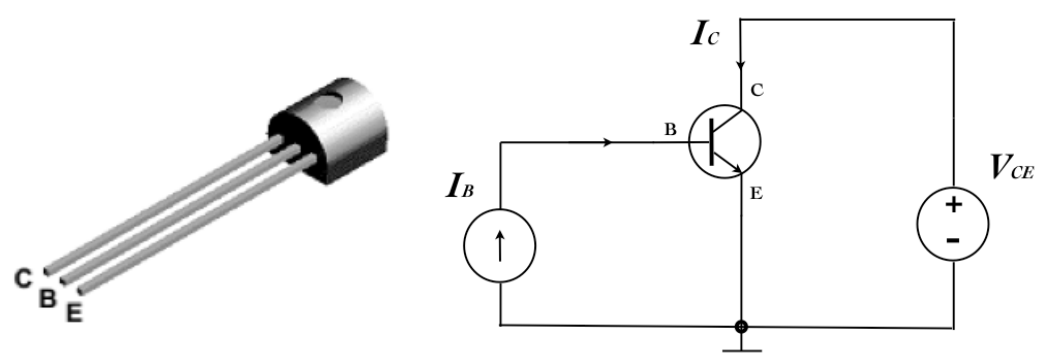

Figura 2. Ilustração de um TBJ NPN típico e o esquema de polarização para detectar os raios $\mathrm{X}$.

\subsection{Irradiações e procedimento de medição}

$\mathrm{O}$ gerador de feixes de raios $\mathrm{X}$ utilizado consistiu em um equipamento hospitalar Polymat Plus 30/50, Siemens, do Centro regional de Ciências Nucleares do Nordeste. Selecionou-se a carga de 
trabalho de 200 mAs e o tempo de exposição de 2000 ms para se efetuar a medição dos seguintes parâmetros: $\mathrm{kVp}$ e CSR. Para este último parâmetro, foi selecionados 3 valores de $\mathrm{kVp}$ : 73, 90 e 117. O procedimento de medição consiste em posicionar o instrumento RXM1407 ou ThinX centralizado sob o feixe de raios X a ser disparado. A leitura do RXM1407 é feita diretamente por um computador conectado ao sistema, e no caso do ThinX seu display apresenta o resultado imediatamente, porém o resultado tem que ser anotado antes do instrumento se desligar automaticamente. A câmara de ionização foi utilizada apenas para comparar os resultados da CSR obtidas com o sistema multi-medição RXM1407. Para fins estatísticos, em todas as avaliações foram feitas ao menos 5 leituras de cada parâmetro ou grandeza para os cálculos das médias e desvios padrões.

Para mensurar o tempo de exposição e o número de pulsos foi utilizado um equipamento de raios $\mathrm{X}$ odontológico Spectro II. O procedimento de medição também é posicionando o instrumento no centro do feixe de raios $\mathrm{X}$ e utilizou-se tempos de exposição de $100 \mathrm{~ms}$.

\section{RESULTADOS}

A Tabela 1 apresenta os resultados da tensão de tubo de raios $X$ obtidos com os instrumentos eletrônicos RXM1407 e ThinX. Observa-se que, para a faixa entre 50kV e 117kV, o sistema multimedição apresenta incertezas relativas menores que $1 \%$, enquanto o instrumento ThinX fornece uma média de 2,3\% para esta amostragem. Verifica-se que o ThinX apresenta valores sistematicamente sub-estimados para a tensão de tubo. Contudo, deve-se salientar que o valor nominal selecionado no equipamento Polymat não necessariamente corresponde ao valor verdadeiro (como se denomina em metrologia), pois o método metrológico de obtenção da kilovoltagem de pico deve ser o da espectrometria com detectores de germânio hiper puro.

Tabela 1. Resultados da tensão de pico medidos com o sistema RXM1407 e ThinX.

\begin{tabular}{|c|c|c|c|c|c|c|c|}
\hline $\begin{array}{c}\text { Tensão (kV) } \\
\text { nominal }\end{array}$ & 50 & 60 & 70 & 81 & 90 & 102 & 117 \\
\hline $\begin{array}{c}\text { ThinX } \\
\text { UNFORS } \\
\text { Erro (\%) }\end{array}$ & 49,7 & 59,0 & 68,7 & 79,0 & 86,9 & 99,0 & 113,7 \\
\hline $\begin{array}{c}\text { RXM1407 } \\
\text { SCIENTS } \\
\text { Erro (\%) }\end{array}$ & 50,4 & $-1,67$ & $-1,90$ & $-2,47$ & $-3,44$ & $-2,94$ & $-2,86$ \\
\hline
\end{tabular}


A Tabela 2 mostra a comparação dos valores da camada semi-redutora (CSR) obtidos com a câmara de ionização e o sistema multi-medição RXM1407. Observa-se que há uma concordância entre os valores com incertezas da ordem de 5\% aproximadamente para a faixa de tensão de tubo entre 70 e $120 \mathrm{kV}$ para esta amostragem de medição.

Tabela 2. Resultados da CSR em mimlímetros de alumínio obtidas com a câmara de ionização 10X5-6 da RADCAL e o sistema multimedição RXM1407 da SCIENTS.

\begin{tabular}{|c|c|c|c|}
\hline Tensão de pico $(\mathrm{kVp})$ & 73 & 90 & 117 \\
\hline Câmara 10X5-6 RADCAL & $2,7 \pm 0,1$ & $3,4 \pm 0,1$ & $4,5 \pm 0,1$ \\
\hline RXM1407 SCIENTS & $2,57 \pm 0,13$ & $3,46 \pm 0,17$ & $4,23 \pm 0,15$ \\
\hline
\end{tabular}

Os resultados da medição do tempo de exposição e do número de pulsos emitidos pelo equipamento de raios X odontológico Spectro II estão apresentados na Tabela 3. Como pode-se observar o sistema RXM1407 fornece o tempo real de exposição aos feixes pulsados de raios X enquanto que o ThinX não consegue diferenciar. De fato o ThinX apresenta o tempo em que o equipamento odontológico fica acionado, embora forneça a quantidade de pulsos. Por outro lado, o multi-medidor RXM1407 além de contar o número de pulsos corretamente, tal sistema fornece como resultado o tempo exato em que o feixe de raios $\mathrm{X}$ esteve presente computando a duração de cada pulso individualmente para somar todos eles ao final da exposição pulsada. A Figura 2 ilustra um exemplo típico de pulsos gerados pelo Spectro II, capturado por um osciloscópio, durante o período em que ele está acionado. De fato, a soma de cada pulso individualmente resulta em um tempo bem inferior ao tempo nominal. Além disso, alguns pulsos de amplitude pequena não são vistos pelo instrumento ThinX como relatado por [7] e exemplificado na Figura 2. Isso ocorre por que os pulsos quando são gerados no início ainda não têm uma amplitude suficiente para sensibilizar os detectores do ThinX, enquanto que o processo eletrônico que utiliza transistores bipolar de junção do RXM1407 permite que estes pulsos sejam vistos pelo sistema multi-medição.

Tabela 3. Resultados do tempo de exposição aos feixes pulsado de raios $\mathrm{X}$.

\begin{tabular}{|c|c|c|c|c|}
\hline \multirow{2}{*}{$\begin{array}{c}\text { Tempo } \\
\text { nominal }(\mathrm{ms})\end{array}$} & \multicolumn{2}{|c|}{ RXM1407 } & \multicolumn{2}{c|}{ ThinX } \\
\cline { 2 - 5 } & Tempo (ms) & $\mathrm{N}^{\circ}$ de pulsos & Tempo (ms) & Pulsos \\
\hline 100 & 53,1 & 9 & $71 \pm 1$ & 5 \\
\hline 100 & 52,1 & 9 & $71 \pm 1$ & 5 \\
\hline 100 & 51,3 & 9 & $104 \pm 1$ & 7 \\
\hline 100 & 51,8 & 9 & $88 \pm 1$ & 6 \\
\hline
\end{tabular}




\begin{tabular}{|l|l|l|l|l|}
\hline 100 & 51,9 & 9 & $87 \pm 1$ & 6 \\
\hline
\end{tabular}
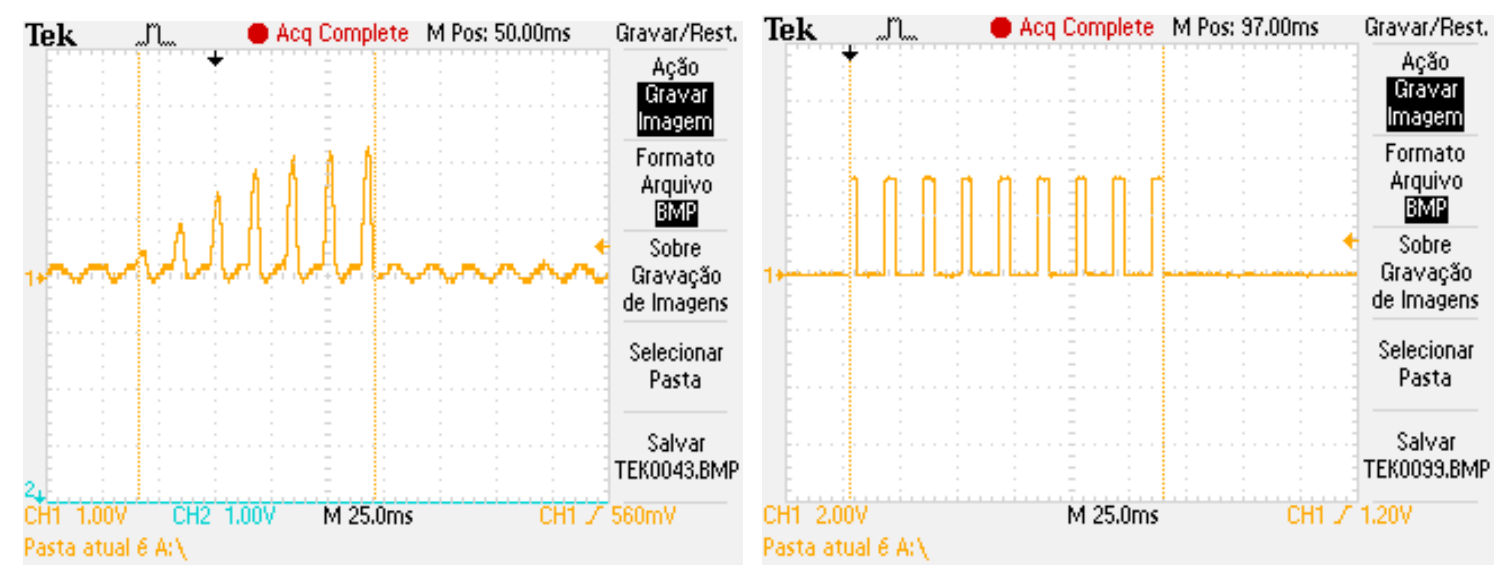

Figura 2. Pulsos de sinais elétricos gerados devido aos fótons de raios $X$ pulsados; Pulsos modulados no sistema de multimedição RXM1407 da SCIENTS.

\section{CONCLUSÕES}

Dois modernos sistemas eletrônicos que são usados para medição de parâmetros de feixes de raios $\mathrm{X}$ utilizados no diagnóstico médico e odontológico foram comparados neste trabalho. Os resultados dos parâmetros $\mathrm{kVp}$ e CSR evidenciam que as incertezas relativas estão abaixo de $1 \%$ e 5\%, respectivamente, ao se mensurar em equipamentos de raios $\mathrm{X}$ diagnósticos convencionais. No entanto, os resultados do equipamento odontológico, que emite pulsos de raios $\mathrm{X}$, tiveram uma divergência significativa pelo fato de cada instrumento ter princípios de funcionamento diferentes. O sistema RXM1407 indica na realidade o tempo real de exposição devido à pulsação da radiação do equipamento de radiologia odontológico. De fato, o sistema de multi-medição RXM1407 utiliza modernas técnicas de medição eletrônica baseadas na avaliação do sinal modulado de transistores bipolar de junção, enquanto que o ThinX mede o tempo total em que equipamento de raios $\mathrm{X}$ pulsados estava ligado.

\section{AGRADECIMENTOS}

À FACEPE pelo suporte financeiro. 


\section{REFERÊNCIAS}

1. ANVISA-MS, Portaria No 453 do Ministério da Saúde, D.O.U., Brasília (1998).

2. C. M. M. Paschoal, D. N. Souza, L. A. P. Santos, Measurement of single scan dose profiles in CT using a commercial photodiode, IEEE Transaction on Nuclear Science, 60, 735-738 (2013).

3. L. A. P. Santos, Cinthia M. S. Magalhães, J. O. Silva, G. G. Araujo, J. A. Filho, E. F. Silva Jr., W. M. Santos, A feasibility study of a phototransistor for the dosimetry of computerized tomography and stereotactic radiosurgery beams, Radiation Measurements, 43, pp. 904-907 (2008).

4. L. A. P. Santos, F. A. Cavalcanti, D. S. Monte, G. G. Araujo, A. N. Alves, G. S. Nobrega, M. A. P. Santos, Techniques for measuring some characteristics of ionizing radiation beams using bipolar junction transistor as a detector, ANIMMA 2013 Proceedings, IEEE Press, Marseille (2013).

5. T. Duncan, Electronics for Today and Tomorrow, John Murray, Dorchester-UK (1997).

6. A. H. Johnston, G. M. Swift, B. G. Rax, Total dose effects in conventional bipolar transistors and linear integrated circuit, IEEE Transaction on Nuclear Science, 41, 2427-2436 (1994).

7. D. B. Silva, Análise comparativa de técnicas de cronometria de raios $\mathrm{X}$ aplicado ao radiodiagnóstico, Dissertação de Mestrado, UFPE, 2013. 